\title{
New MRI Findings in Fukuyama Congenital Muscular Dystrophy: Brain Stem and Venous System Anomalies
}

\author{
(D) A. Hirasawa-Inoue, (D) N. Sato, (D) Y. Shigemoto, (D). Kimura, (D) A. Ishiyama, (DE. Takeshita, (D) M. Mori-Yoshimura, (D). Oya, \\ (D) Y. Takahashi, (D) H. Komaki, (D) H. Matsuda, and (D) M. Sasaki
}

\begin{abstract}
BACKGROUND AND PURPOSE: Leptomeningeal glioneuronal heterotopia of the brain stem and cerebral migration abnormality were pathologically reported in Fukuyama congenital muscular dystrophy, but the radiologic assessments of the brain stem and cerebral venous system (which may be involved in the development of the anomaly) were insufficient. Here, we evaluated the brain stem and cerebral veins on MR imaging in patients with Fukuyama congenital muscular dystrophy.
\end{abstract}

MATERIALS AND METHODS: We retrospectively reviewed the MR imaging findings of 27 patients with Fukuyama congenital muscular dystrophy. We visually assessed the hypoplasia, superficial structures, and signal intensity of the brain stem on T2WI, FLAIR, and double inversion recovery images and the cerebral, superficial, and deep veins with and without hemorrhage on T2WI and SWI.

RESULTS: Brain stem fluffy structures were seen in $96.3 \%$ of the cases on T2WI. Superficial high signal intensity on T2WI and FLAIR images was seen in $96.3 \%$ and $92.6 \%$, respectively. Abnormally located superficial vessels beneath the cortex were seen in $11.1 \%$ on T2WI. Hypoplasia of the superficial cerebral veins was noted in all patients who underwent SWI. Dilated and tortuous subependymal veins were seen in $40.0 \%$ on SWI. Hemorrhages were seen in $11.1 \%$ on T2WI and in $60.0 \%$ on SWI.

CONCLUSIONS: Superficial brain stem structural and signal abnormalities would be useful MR imaging findings to diagnose Fukuyama congenital muscular dystrophy as well as venous system abnormalities. Clinicians must keep in mind that this disease has a high risk of hemorrhage.

ABBREVIATIONS: DIR = double inversion recovery; FCMD = Fukuyama congenital muscular dystrophy

$\mathbf{P}$ atients with Fukuyama congenital muscular dystrophy (FCMD) have generalized muscle weakness, hypotonia, and developmental delay beginning in early infancy. Characteristic brain MR imaging findings and high serum creatine kinase levels facilitate a clinical diagnosis of FCMD. The characteristics of FCMD as shown by brain MR imaging are cortical dysplasia (polymicrogyria or pachygyria), cerebellar cysts, cerebellar cortical dysplasia, white matter abnormality, and brain stem hypoplasia. ${ }^{1-3}$

Received January 6, 2020; accepted after revision April 3.

From the Departments of Child Neurology (A.H.-I., A.I., E.T., H.K., M.S.), Radiology (N.S., Y.S., Y.K.), and Neurology (M.M.-Y., Y.O., Y.T.), National Center Hospital, Translational Medical Center (H.K.), and Integrative Brain Imaging Center (H.M.), National Center of Neurology and Psychiatry, Tokyo, Japan.

Please address correspondence to Noriko Sato, MD, PhD, Department of Radiology, National Center Hospital, National Center of Neurology and Psychiatry, 4-1-1 Ogawahigashi-cho, Kodaira-shi, Tokyo, Japan; e-mail: snoriko@ncnp.go.jp

Indicates article with supplemental on-line table.

http://dx.doi.org/10.3174/ajnr.A6577
We recently noticed that many cases of FCMD showed abnormal signals along the brain stem surface on T2WI and FLAIR images. Pathologic studies disclosed leptomeningeal glioneuronal heterotopia, abnormal courses of the corticospinal tracts, and neuronal loss and degeneration in the brain stem. ${ }^{4,5}$ However, radiologic assessments of the brain stem have not been published, and we have treated patients with FCMD who showed cerebral vascular abnormalities in both deep and superficial vessels with and without hemorrhage on T2WI or SWI. Cortical dysplasia is known to be caused by an overmigration of neurons into the subarachnoid space through breaches in the glia limitans, ${ }^{6}$ and abnormal changes of the vascular endothelial cells have been observed in biopsied muscle samples. ${ }^{7,8}$ Abnormal vessels running beneath the cerebral cortex of patients with FCMD were observed on T2WI. ${ }^{1}$ To the best of our knowledge, there is no published report of the venous system anomalies or hemorrhages in FCMD that are most detectable by SWI. We conducted the present study to evaluate brain MR imaging findings in patients with FCMD, including the findings of the brain stem and cerebral venous system. 


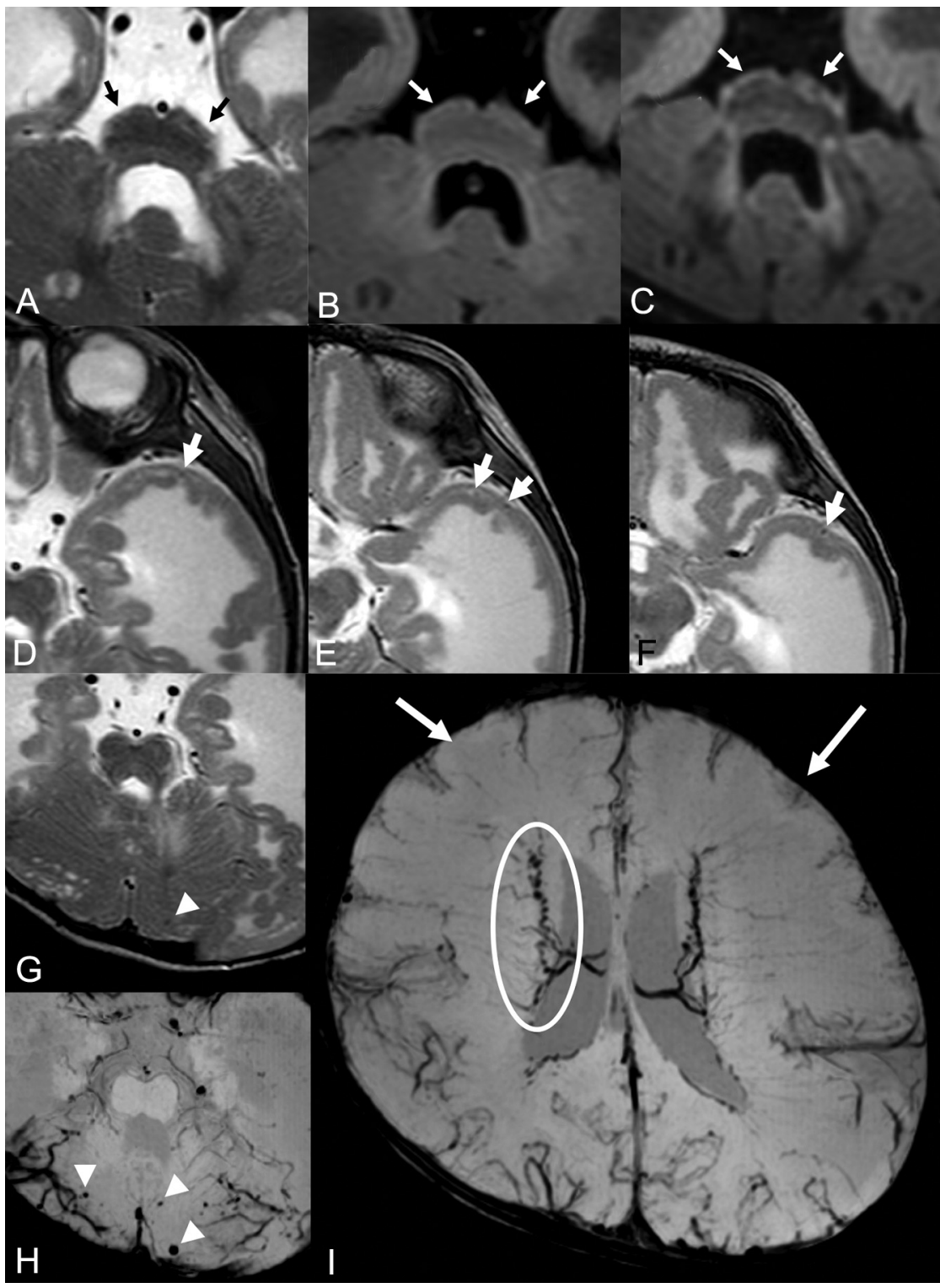

FIG 1. Brain MR imaging of patient 15. Brain stem images on T2WI (A), FLAIR (B), and DIR (C). There is a fluffy structure on T2WI (A, black arrows) and high intensity of the hypoplastic pontine surface on the FLAIR image $(B$, white arrows). High signal intensity along the brain stem surface on the DIR image is recognized more clearly than on the T2WI or FLAIR image ( $C$, white arrows). T2Wls of the left temporal lobe $(D-F)$ show lissencephalic and the abnormally located cortical superficial vessels running through the superficial layer of the lissencephalic cortex (white arrows). Microhemorrhages were seen in the cerebellar cyst on T2WI $(G)$ and SWI $(H)$. More microhemorrhages are recognizable on the SWI than on T2WI (white arrowheads). Cerebral SWI (I) shows hypoplasia of a superficial cerebral vein in both frontal lobes (white arrows) and dilated and tortuous subependymal veins (white circle).

\section{MATERIALS AND METHODS}

We retrospectively reviewed the MR imaging findings of the 53 patients who were clinically and genetically diagnosed with FCMD at our hospital from 2006 through 2018. We excluded 26 patients who did not have both axial T2WI and FLAIR images as brain MR imaging because axial T2WI and FLAIR images are required for the evaluation of the brain stem. We examined a final total of 27 patients: 17 males and 10 females (age range, 5 months to 23 years; median, 18 months; mean, 6 years). Axial 2D- or 3D-T1WI, axial T2WI, and FLAIR images were obtained for all 27 patients. Six patients also had 3D double inversion recovery (DIR) images, and 5 patients had SWI findings. This retrospective study was approved by the institutional review board of our hospital, and the need for patient informed consent was waived.

We evaluated the presence/absence of brain stem hypoplasia (ie, a small and flattened ventral portion at the level of the middle cerebellar peduncle, a fluffy brain stem surface on T2WI, and high signals of the brain stem surface on T2WI, FLAIR, and DIR images). We also evaluated the presence or absence of abnormal vessels running just beneath the cortical surface on T2WI, hemorrhage on T2WI and SWI, and whether the cerebral superficial and deep veins are hypoplastic or dilated on SWI. We assessed other MR imaging findings: cerebellar cysts, cerebral cortical dysplasia (polymicrogyria or pachygyria), and white matter abnormal high signals (patchy/spotty or diffuse) on T1WI, T2WI, and FLAIR images. Two neuroradiologists (Y.K. and N.S. with 14 and 30 years of experience in neuroradiology, respectively) independently evaluated all images. The differences were resolved by consensus.

\section{RESULTS}

The clinical and MR imaging findings for all 27 patients are summarized in the On-line Table. All patients showed brain stem hypoplasia (Figs 1 and 2). We observed a fluffy brain stem surface and mild high signal intensity along the surface of a hypoplastic pons on T2WI in 26 patients $(96.3 \%)$ and FLAIR images in 25 patients (92.6\%) (Figs $1 A$, $-B$ and $2 A,-B)$. The DIR images of all 6 patients showed high signal intensity along the brain stem surface (Fig 1C), and the high signal intensity was recognized more clearly on the DIR images than on the T2WI and FLAIR images.

Abnormally located superficial vessels beneath the cortex were observed in 3 patients (11.1\%). The vessels ran through, inside, or beneath the lissencephalic cortex, and they were recognizable especially in the temporal lobes (Figs $1 D-F$ and $2 C-E$ ). Hypoplasia of superficial cerebral veins around the frontal lobes was noted in all 5 patients who had SWI findings (Figs $1 I$ and 3C, $-D)$. Dilated and tortuous subependymal veins were identified in 2 of these 5 patients $(40.0 \%)$ on SWI (Figs $1 I$ and $3 C,-D$ ). 


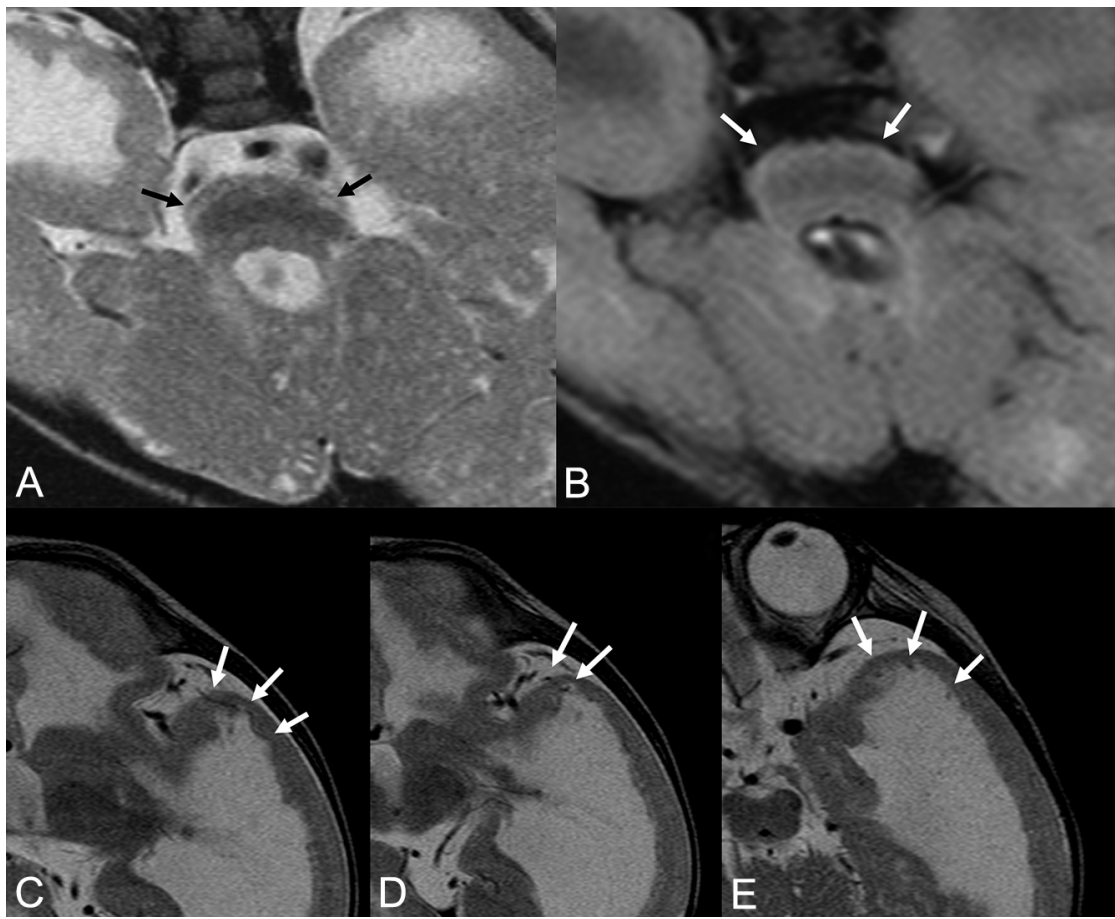

FIG 2. Brain stem $(A$ and $B)$ and left temporal $(C-E)$ MRIs of patient 20. A brain stem T2WI $(A)$ shows a fluffy structure and high intensity of the hypoplastic pontine surface (black arrows). The FLAIR image (B) detects high intensity of the pontine surface (white arrows). Left temporal T2WIs $(C-E)$ show lissencephaly, which contains abnormally located superficial cortical vessels running through and beneath the cortex (white arrows).

Hemorrhages were detected on T2WI in 3 of the 27 patients $(11.1 \%)$ and on SWI in 3 of the 5 patients (60.0\%). Patient 7 showed microhemorrhages in the cerebral and cerebellar cysts on SWI. Numerous microhemorrhages were seen in the cerebellar cysts in patient 15 on T2WI and SWI (Fig $1 G,-H$ ). Patient 23 exhibited a hemorrhage in the white matter around the occipital horn of the left lateral ventricle on T2WI and SWI. Patient 25 showed a hemorrhage in the white matter of the frontal lobe on T2WI (Fig 4).

All 27 patients had a degree of cerebral dysplasia; the degrees varied among the patients. Polymicrogyria was recognized in all 27 patients. The frontal lobe was involved in all patients, and the temporoparietal lobes were affected in some patients. Pachygyria that involved mainly the temporo-occipital lobes was also seen in 4 patients. All 27 patients had T1 low signal and T2 high signal in the white matter (Figs 1, 2, and 4). The signal extent varied among the patients. Cerebellar intraparenchymal cysts located in the peripheral hemispheres were identified in 26 patients.

\section{DISCUSSION}

We assessed the brain stem and cerebral venal abnormalities and hemorrhage in patients with FCMD on brain MR imaging, including DIR and SWI. Fluffy structures around the pons and high signal intensity along the surface of hypoplastic pons were confirmed in $96.3 \%$ of the patients on T2WI. On SWI, hypoplasia of superficial cerebral veins around the frontal lobes was noted in all patients, and dilated and tortuous subependymal veins were seen in $40.0 \%$. Hemorrhages were seen in $11.1 \%$ on T2WI and $60 \%$ on SWI. Thus, the identification of brain stem marginal abnormal findings and venous system anomalies could help diagnose FCMD. Our findings also indicate that FCMD presents a high risk of hemorrhage as a complication.

Cerebral cortical developmental abnormalities are well-known in FCMD. Cortical dysplasia is caused by the overmigration of neurons into the subarachnoid space through breaches in the glia limitans. ${ }^{6}$ FCMD belongs to a group of $\alpha$-dystroglycanopathies; $\alpha$-dystroglycan is important for normal basement membrane formation and neuronal migration. It was suggested that a dystroglycannull brain loses its high-affinity binding to the extracellular matrix protein laminin and shows discontinuities in the pial surface basal lamina. ${ }^{9}$ In addition, a FKTN gene defect may result in functional disruption through the hypoglycosylation of both neuronal and glial $\alpha$-dystroglycan. ${ }^{10}$

Similar pathology is observed in the brain stem as well as the cerebral cortex in FCMD. Leptomeningeal glioneuronal heterotopia and an aberrant pyramidal tract in the brain stem of patients with FCMD have been described. ${ }^{11}$ In another study, the cases of FCMD had a band of gliotic tissue along the surface of the brain stem, and this made the glia limitans thicker than in healthy control subjects. Pontine nuclei exhibiting some neuronal clusters that protruded into the subarachnoid space at the ventrolateral pontine surface were also observed; this parenchymal protrusion extended ventromedially, tangential to the pontine surface. ${ }^{5}$ We speculate that the fluffy structures and high signal intensity along the brain stem surface on T2WI, FLAIR, and DIR images reflect leptomeningeal glioneuronal heterotopia, a thick glia limitans, or an extrapial protrusion of glial tissue.

DIR images suppress signals from both white matter and CSF and may enhance visualization of abnormal features at the graywhite matter interface; then, we could see brain stem abnormality more clearly.

We also observed both abnormally located cortical superficial vessels running through, inside, or beneath the lissencephalic cortex and hypoplasia of superficial cerebral veins around the frontal lobes. Abnormal vessels running beneath the cerebral cortex on T2WI were reported, ${ }^{1}$ and it was confirmed pathologically that there were many small vessels that entered the cortex through the breaches in the glia limitans. ${ }^{6}$ However, the dilated and tortuous subependymal veins revealed in the present study had not been reported previously. Hypoplasia of superficial cerebral veins around frontal lobes would be a secondary consequence of overmigration. As a result, a subependymal vein may be dilated. 


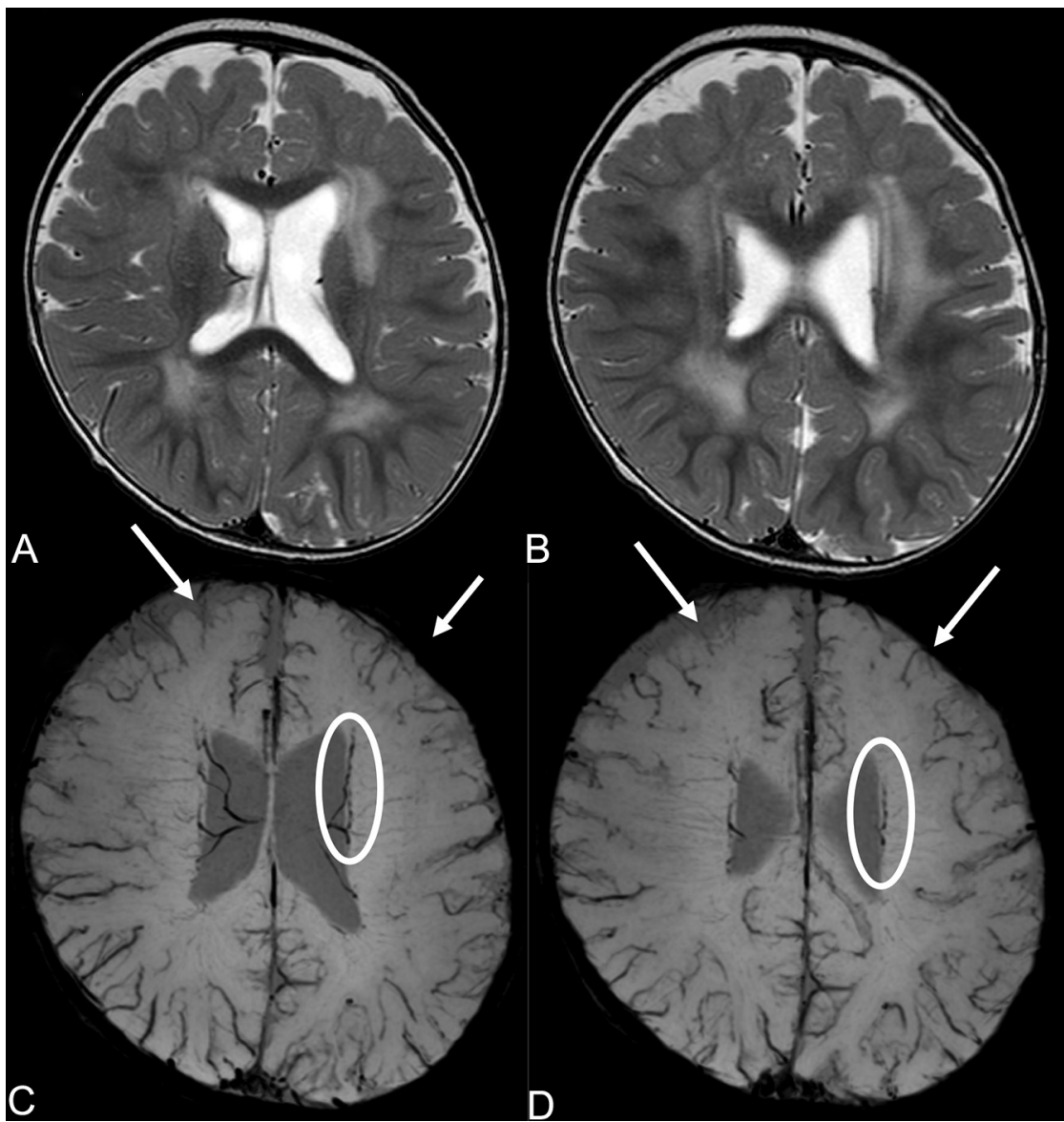

FIG 3. Cerebral MRIs of patient 13. T2WIs ( $A$ and $B$ ) show polymicrogyria and diffuse high signal in the white matter. SWIs ( $C$ and $D$ ) reveal hypoplasia of superficial cerebral veins in the frontal lobe (white arrows) and meandering subependymal veins (white circles).

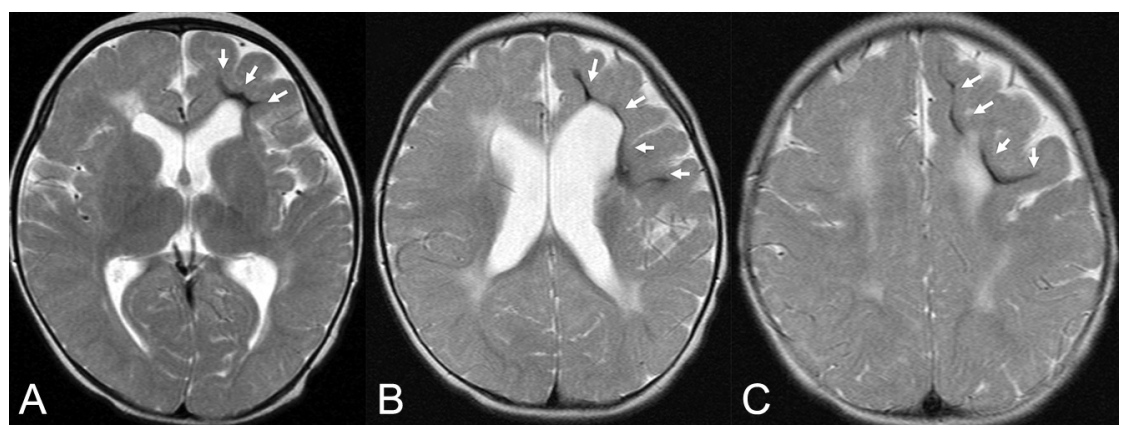

FIG 4. Cerebral MRIs of patient 25. There are hemorrhages in the deep and subcortical white matter of the atrophic left frontal lobe (white arrows) on T2WIs. The location of the hemorrhage in this patient is different from that of the subependymal hemorrhage in a premature neonate.

On the other hand, it is possible that venous abnormalities can be a primary result of a dysfunction of $\alpha$-dystroglycan. In a muscle pathology study of patients with FCMD, vascular changes, including replication of the basement membrane, blisterlike swelling of endothelial cells, and platelet adhesion and aggregation on small blood vessels, were observed. ${ }^{8}$ It was reported that dystroglycan was expressed in vascular endothelial cells in the human brain and that endothelial dystroglycan plays a role in angiogenesis. $^{12,13}$ In addition, intersegmental vessels and distorted eye vasculature were seen in a FKTN morphant. It is suspected that the disruption of angiogenesis was a primary result caused directly by the knockdown of FKTN and dystroglycan. ${ }^{7}$ We speculated that not only would hypoplasia of superficial cerebral veins result in the enlargement of subependymal veins but vulnerable brain vessels would also be related to dilated subependymal veins.

Hemorrhages can occur as a result of the vulnerability of brain vessels and congestion due to hypoplasia of superficial cerebral veins. Assessment of brain vessel abnormalities and the presence/absence of hemorrhage will contribute to the management of patients with FCMD.

There are a few limitations to this study. Although an earlier study indicated a relationship between motor function and brain stem abnormality, ${ }^{14}$ we were not able to evaluate the relationship between the maximum motor function and brain stem dysplasia or venous abnormalities in our patient series because some of the 27 patients came to our hospital only for the diagnosis and we could not follow them to the age at which they reached the maximum motor function. In addition, there were only 5 patients who had SWI findings, and SWI is the most suitable sequence to precisely assess the presence and severity of vessel anomalies and hemorrhages. Evaluations of SWI of patients with FCMD would provide more information.

\section{CONCLUSIONS}

The results of our retrospective analyses of the imaging of 27 patients with FCMD demonstrated the fluffy structure around the pons and high signal intensity along the surface of a hypoplastic brain stem on T2WI/FLAIR/DIR images. These findings can be useful in the diagnosis of FCMD. Hemorrhage and abnormalities of cerebral superficial and subependymal veins were also highly meaningful MR imaging findings. Clinicians must keep in mind that patients with FCMD are at risk of incurring a hemorrhage, and follow-up MR imaging would be useful to manage these patients. 


\section{REFERENCES}

1. Aida N, Tamagawa K, Takada K, et al. Brain MR in Fukuyama congenital muscular dystrophy. AJNR Am J Neuroradiol 1996;17:60513 Medline

2. Aida N. Fukuyama congenital muscular dystrophy: a neuroradiologic review. J Magn Reson Imaging 1998;8:317-26 CrossRef Medline

3. Clement E, Mercuri E, Godfrey C, et al. Brain involvement in muscular dystrophies with defective dystroglycan glycosylation. Ann Neurol 2008;64:573-82 CrossRef Medline

4. Itoh M, Houdou S, Kawahara H, et al. Morphological study of the brainstem in Fukuyama type congenital muscular dystrophy. Pediatr Neurol 1996;15:327-31 CrossRef Medline

5. Saito $\mathrm{Y}$, Kobayashi M, Itoh M, et al. Aberrant neuronal migration in the brainstem of Fukuyama-type congenital muscular dystrophy. J Neuropathol Exp Neurol 2003;62:497-508 CrossRef Medline

6. Nakano I, Funahashi M, Takada K, et al. Are breaches in the glia limitans the primary cause of the micropolygyria in Fukuyamatype congenital muscular dystrophy (FCMD)? Pathological study of the cerebral cortex of an FCMD fetus. Acta Neuropathol 1996; 91:313-21 CrossRef Medline

7. Wood AJ, Muller JS, Jepson CD, et al. Abnormal vascular development in zebrafish models for fukutin and FKRP deficiency. Hum Mol Genet 2011;20:4879-90 CrossRef Medline
8. Sugino S, Miyatake M, Ohtani Y, et al. Vascular alterations in Fukuyama type congenital muscular dystrophy. Brain Dev 1991; 13:77-81 CrossRef Medline

9. Moore SA, Saito F, Chen J, et al. Deletion of brain dystroglycan recapitulates aspects of congenital muscular dystrophy. Nature 2002;418:422-25 CrossRef Medline

10. Saito Y, Yamamoto T, Mizuguchi M, et al. Altered glycosylation of alpha-dystroglycan in neurons of Fukuyama congenital muscular dystrophy brains. Brain Res 2006;1075:223-28 CrossRef Medline

11. Fukuyama $Y$, Osawa M, Suzuki H. Congenital progressive muscular dystrophy of the Fukuyama type: clinical, genetic and pathological considerations. Brain Dev 1981;3:1-29 CrossRef Medline

12. Yamamoto T, Shibata N, Kanazawa M, et al. Localization of laminin subunits in the central nervous system in Fukuyama congenital muscular dystrophy: an immunohistochemical investigation. Acta Neuropathol 1997;94:173-79 CrossRef Medline

13. Hosokawa H, Ninomiya H, Kitamura $Y$, et al. Vascular endothelial cells that express dystroglycan are involved in angiogenesis. $J$ Cell Sci 2002;115:1487-96 Medline

14. Kato I, Osawa M, Murasugi S, et al. Neuroimaging morphological study of brain stem and cerebellum in cases of Fukuyama type congenital muscular dystrophy. Journal of Tokyo Women's Medical University 1998;68:772-78 